\title{
Review of: "Factors associated with and socioeconomic inequalities in breast and cervical cancer screening among women aged 15-64 years in Botswana"
}

\author{
MANUEL EMILIO COLOME HIDALGO ${ }^{1}$ \\ 1 Instituto Tecnológico de Santo Domingo
}

Potential competing interests: The author(s) declared that no potential competing interests exist.

This is an observational study based on Noncommunicable Diseases survey data in Botswana conducted in 3 cities and towns, 15 urban villages, and 15 rural areas in 2016. The study evaluated the factors associated with socioeconomic inequalities in breast and cervical cancer screening among women aged 15-64 years.

The outcome variables used by the authors were: Breast and cervical cancer screening. The authors used age, education, socioeconomic status, employment and wealth status, marital status, area of residence as explanatory variables, which are part of the dimensions of inequality established in the framework of social determinants of the World Health Organization (WHO) (See: https://www.who.int/health-topics/socialdeterminants-of- health).

The authors performed univariate/bivariate analyses and logistic regression models to analyze the data and to assess the association between the explanatory and outcome variables, using adjusted probability ratios and a 95\% confidence interval. Health inequality analysis included concentration curves, estimation of the concentration index, and the achievement index. The main finding in this study was that women from poorer households had lower odds of detection of cervical and breast cancer compared with women in the highest wealth quintile, highlighting the role of socioeconomic inequalities in cancer detection. Despite the limitations of the study design, the authors highlight two important limitations: (a) The impossibility of establishing causal inference from the findings and (b) The findings in the study should be interpreted with caution, the study analyzed data from a non-representative sample from Botswana. Thus, the research findings are not generalizable. Regardless of these limitations, there are some concerns with this study including the following:

1. There are limitations inherent to the design and implementation of the Botswana Noncommunicable Diseases Survey 2016, which are not explored and could have a significant impact on the conclusions of this study. Placing a reference to the survey's technical note would allow readers to get a complete idea 
about the biases and limitations related to the data source, this being a key element when evaluating the quality and scope of the data.

2. The disaggregation of the level education category "Primary or less" (41.2\%) could increase the analysis of inequalities and provide statistically significant data.

3. Study estimates of women reporting cervical (62\%) and breast (6\%) screening are likely to be affected by age groups, and these in turn by recommendations and policies of the prevention programs of the Ministry of Health and Welfare of Botswana. This approach explains why the chances of detection of cervical and breast cancer have increased with age. The low detection rate of breast cancer at a general level is impressive; the authors could discuss more details about this finding by comparing it with official national statistics and delving deeper into possible explanations for this phenomenon. Why have cervical cancer screening recommendations received more attention compared to breast cancer in Botswana? What makes Botswana different from other countries with similar health systems and income?

4. The social vulnerability condition that retirement status implies is another factor that could explain why this group (despite being older) showed a lower probability of cancer detection than public sector employees. This conclusion reaffirms the importance of studying other determinants of access to the health services involved.

5. The descriptive analysis of inequalities should include simple measures (i.e. absolute and relative inequalities) and complex measures (i.e. slope inequality index). WHO has a standardized guide for reporting inequalities focused on low- and middle-income countries. The use of simple and complex inequality measurements helps readers to have a big picture of health inequalities.

(See: https://www.who.int/data/gho/health-equity/handbook).

This study (highly recommended) is relevant as it provides more evidence about the importance of socioeconomic determinants and health inequalities in 15-64 women. The findings of this study could contribute to the development of public health policies aimed at closing inequalities gaps to achieve universal health, not only in Botswana but also in other low and middle-income countries. 